\title{
UNIFORM SPACES, SPANIER QUASITOPOLOGIES, AND A DUALITY FOR LOCALLY CONVEX ALGEBRAS
}

\author{
EDUARDO J. DUBUC and HORACIO PORTA
}

(Received 19 April 1979)

Communicated by $\mathbf{R}$. Street

\begin{abstract}
Gelfand-type duality results can be obtained for locally convex algebras using a quasitopological structure on the spectrum of an algebra (as opposed to the topologies traditionally considered). In this way, the duality between (commutative, with identity) $C^{*}$-algebras and compact spaces can be extended to pro- $C^{*}$-algebras and separated quasitopologies. The extension is provided by a functional representation of an algebra $A$ as the algebra of all continuous numerical functions on a quasitopological space. The first half of the paper deals with uniform spaces and quasitopologies, and has independent interest.
\end{abstract}

1980 Mathematics subject classification (Amer. Math. Soc.): 46 H 05, 46 J 10, 18 B 30, 18 D 20, $54 \mathrm{E} 15$.

It was shown in Dubuc and Porta (1971) that duality results of the type Gelfand obtained for $C^{*}$-algebras can be formulated in (and proved with) the machinery of enriched category theory. Given a class $\mathscr{A}$ of topological algebras, in order to follow this principle a basic starting point is to furnish the class $\mathscr{A}$ with an enriched category structure over a convenient (cartesian closed) base category of objects of a topological nature, and in such a way that $\mathscr{A}$ be cotensored. That is, in addition to providing the set of morphisms between algebras with structures of topological nature, $\mathscr{A}$ has to be sufficiently complete to ensure the existence of left adjoints for these enriched representable functors.

With $\mathscr{A}$ the class of locally convex topological algebras, we showed in Dubuc and Porta (1971) how to do this with the category of Kelley spaces as base category. Given a Kelley space $X$ and an algebra $A$ in $\mathscr{A}$, the algebra $\mathscr{C}(X, A)$ of continuous functions on $X$ with values in $A$ is a natural candidate for the cotensor of $X$ with $A$, if we provide $\mathscr{C}(X, A)$ with the uniform convergence of compact subsets of $X$. 
Briefly, we should expect to have a bijection (natural in $B \in \mathscr{A}$ ):

$$
\frac{X \longrightarrow \mathscr{A}(B, A), \text { continuous }}{B \longrightarrow \mathscr{C}(X, A), \text { morphism in } \mathscr{A}}
$$

where $\mathscr{A}(B, A)$ denotes the set of morphisms $B \longrightarrow A$ in $\mathscr{A}$, conveniently topologized. This can be done (see Dubuc and Porta (1971)) if $\mathscr{A}$ is the category of locally convex algebras with morphisms from $B$ to $A$ all linear multiplicative maps $B \longrightarrow A$ that have continuous restrictions to the compact subsets of $B$. The topology on $\mathscr{A}(B, A)$ that makes (1) a bijection is the $k$-ation of the compactopen topology. We are forced to consider non-continuous maps since from the continuity of $X \longrightarrow \mathscr{A}(B, A)$ only follows the continuity of $B \longrightarrow \mathscr{C}(X, A)$ on compact sets. Clearly, allowing these non-continuous maps as morphisms of $\mathscr{A}$ identifies in $\mathscr{A}$ different locally convex structures on a given algebra when they have the same compact sets. That is, isomorphisms in $\mathscr{A}$ do not guarantee that the locally convex structures are topologically equivalent. Several attempts have been made to provide the set of continuous algebraic homomorphisms with some topology in order to obtain extensions of the classical Gelfand duality for commutative $C^{*}$-algebras (see, for example, Michael (1952), Hsia (1959)). But all such efforts present irregularities that stem from the fact that, if we accept only the continuous algebraic morphisms, then (1) is not a bijection for the topologies so far considered on $\mathscr{A}(B, A)$.

We believe that, in fact, this will be the case for any natural choice of topology on $\mathscr{A}(B, A), A, B \in \mathscr{A}$. However, even if no topology can be found for $\mathscr{A}(B, A)$, we know which functions $X \longrightarrow \mathscr{A}(B, A)$ we want to accept as continuous: exactly those that arise from continuous morphisms $B \longrightarrow \mathscr{C}(X, A)$. In particular, when $K$ is compact, we should have a bijection:

$$
\frac{K \longrightarrow \mathscr{A}(B, A), \text { continuous }}{B \longrightarrow \mathscr{C}(K, A), \text { continuous algebraic morphisms }}
$$

It so happens that the concept of a set with a family of admissible ( $=$ 'continuous') maps from each compact space is a structure of topological nature considered by Spanier (1959), and called a quasitopology. Since quasitopological spaces form a cartesian closed category $\mathscr{Q}$, it serves our purpose: we can expect to be able to enrich $\mathscr{A}$ over $\mathscr{L}$ (that is, make each $\mathscr{A}(B, A)$ into a quasitopological space) in such a way that (1) is a bijection making $\mathscr{A}$ a cotensored category appropriate for duality.

In this paper we carry out this program to obtain an exact topological equivalence between pro- $C^{*}$-algebras $B$ and algebras of continuous functions over Spec $(B)$, the spectrum of $B$ (a quasitopological space). Not surprisingly, this duality is essentially contained in the work of Michael (1952), well before the 
invention of quasitopologies. Analysing when $\operatorname{Spec}(B)$ is actually a topological space we can easily get results in Michael (1952).

As the Gelfand theory identifies the dual category of commutative $C^{*}$-algebras with 1 (and continuous algebraic homomorphisms, which are all the algebraic homomorphisms in this case) to be the category of compact spaces, we identify here the dual of the category of Pro- $C^{*}$-algebras (and continuous algebraic homomorphisms) to be the category $\mathscr{Q}_{2}$ of separated quasitopologies. These are the quasitopological spaces with enough real continuous functions to separate points. They satisfy a Hausdorff-type separation axiom and form a full reflexive subcategory of $\mathscr{Q}$. The reflexion follows from our work below. $\mathscr{Z}_{2}$ is also cartesian closed.

We observe that the success of our program depends only on the uniform structure associated to a locally convex algebra. Therefore, in order to simplify the exposition and in view of other possible applications, we develop the basic machinery at that level in Sections 1 and 2, and show that uniform spaces with uniformly continuous maps form a category that can be enriched over 2 , to become a cotensored 2 -category. Then, in Sections 3 and 4 we apply this machinery to the case of locally convex algebras.

For the reader unfamiliar with category theory we always include an explanation of categorical statements and terms.

\section{Contents}

\$0 Background

$\S 1$ Relationship between uniform spaces and Spanier's quasitopologies

$\$ 2$ The enrichment of the category of uniform spaces over the category of quasitopologies

$\$ 3$ Liftings of the enrichment

$\S 4$ The enriched category of locally convex algebras

\section{Background}

In this section we will recall the notion of quasitopology (due to Spanier (1963)) and state some basic properties needed further on. Proofs will be provided only when they cannot be found elsewhere. The reader is referred to Spanier (1959, 1963), Clark (1969) and Eilenberg (1969).

(0.1) By a compact space we mean a topological space satisfying both the HeineBorel converging property and the Hausdorff separation axiom. 
(0.2) A quasitopological space is a set $X$ together with, for each compact space $K$, a set of admissible maps $K \stackrel{\alpha}{\longrightarrow} X$, such that the following axioms (Q1), (Q2) and (Q3) are satisfied:

(Q1) All maps $1 \longrightarrow X$ are admissible (where 1 denotes the compact space with one point).

(Q2) (Pre-sheaf axiom.) If $W$ and $K$ are compact, $W \stackrel{f}{\longrightarrow} K$ is continuous and $K \stackrel{\alpha}{\longrightarrow} X$ is admissible, then $W \stackrel{f}{\longrightarrow} K \stackrel{\alpha}{\longrightarrow} X$ is also admissible.

(Q3) (Finite covering sheaf axiom.) If $W_{i} \stackrel{f_{i}}{\longrightarrow} K, i=1,2, \ldots, n$, is a finite subjective family of continuous maps with $W_{i}$ and $K$ compact and all composites $W_{i} \stackrel{f_{i}}{\longrightarrow} K \stackrel{\alpha}{\longrightarrow} X$ admissible, then $K \stackrel{\alpha}{\longrightarrow} X$ is also admissible.

(0.3) A function $X \stackrel{f}{\longrightarrow} Y$ between two quasitopological spaces is continuous if $K \stackrel{\alpha}{\longrightarrow} X \stackrel{f}{\longrightarrow} Y$ is admissible for each $K \stackrel{\alpha}{\longrightarrow} X$ admissible.

We shall denote by $\mathscr{Q}$ the category of quasitopological spaces with continuous maps. Observe that compact spaces $W$ are canonically quasitopological spaces with all continuous $K \stackrel{\alpha}{\longrightarrow} W$ as admissible maps.

(0.4) The product of two quasitopological spaces $X$ and $Y$ is the set $X \times Y$ with admissible maps those $C \stackrel{\gamma}{\longrightarrow} X \times Y$ that are admissible in each coordinate (that is, such that $C \stackrel{\gamma}{\longrightarrow} X \times Y \stackrel{\pi_{1}}{\longrightarrow} X$ and $C \stackrel{\gamma}{\rightarrow} X \times Y \stackrel{\pi_{2}}{\rightarrow} Y$ are admissible where $\pi_{1}$ and $\pi_{2}$ are the projections). It is easy to see that any admissible maps $C \stackrel{\gamma}{\longrightarrow} X \times Y$ factors through an admissible map of the form $K \times W \stackrel{\alpha \times \beta}{\longrightarrow} X+Y$ with $K \stackrel{\alpha}{\longrightarrow} X$ and $W \stackrel{\beta}{\longrightarrow} Y$ admissible.

(0.5) The category 2 has all limits. In fact, suppose that a functor $\Gamma \longrightarrow \mathscr{Q}$, $\lambda \longrightarrow X_{\lambda}$ is given. A diagram $X \stackrel{\pi_{\lambda}}{\longrightarrow} X_{\lambda}$ is a limit diagram for this functor if $X$ is the set $X=\lim _{\leftarrow} X_{\lambda}$ (with $\pi_{\lambda}$ the canonical projections) and $K \stackrel{\alpha}{\longrightarrow} X$ is admissible if and only if $\pi_{\lambda} \circ \alpha$ is admissible for all $\lambda$.

(0.6) The category $\mathscr{Q}$ has all colimits. Given a functor $\Gamma \longrightarrow \mathcal{Q}, \lambda \mapsto X_{\lambda}$, a diagram $X_{\lambda} \stackrel{g_{\lambda}}{\longrightarrow} X$ is a limit diagram for it if $X$ is the set $X=\underset{\rightarrow}{\operatorname{colim}} X_{\lambda}$ (with $g_{\lambda}$ the canonical inclusions) and $K \stackrel{\alpha}{\longrightarrow} X$ is admissible if and only if there exists a finite surjective family of continuous functions $K_{i} \stackrel{f_{i}}{\longrightarrow} K$ and admissible maps $K_{i} \stackrel{f_{i}}{\longrightarrow} X_{\lambda_{i}}$ making the following diagram commutative.

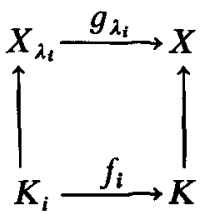


(0.7) A sub-quasitopology or subspace-quasitopology is, by definition, an injective initial map. In other words, it is a continuous injective function $X \stackrel{i}{\longrightarrow} Y$ such that a function $Z \stackrel{g}{\longrightarrow} X$ is continuous if and only if the composite $Z \stackrel{g}{\longrightarrow} X \stackrel{i}{\longrightarrow} Y$ is continuous. We say that $X$ has the initial quasitopology induced by $Y$ and $i$. Given a quasitopological space $Y$ and an injective function $X \stackrel{i}{\longrightarrow} Y$ from a set $X$, the maps $K \stackrel{\alpha}{\longrightarrow} X$ for which $i \circ \alpha$ is admissible define a quasitopology on $X$ which is initial for $i$.

(0.8) A quotient quasitopology is a surjective final map. In other words, it is a continuous surjective function $B \stackrel{f}{\longrightarrow} X$ such that a function $X \stackrel{g}{\longrightarrow} Z$ is continuous if and only if the composite $Y \stackrel{f}{\longrightarrow} X \stackrel{g}{\longrightarrow} Z$ is continuous. Given a quasitopological space $Y$ and a surjective function $Y \stackrel{f}{\longrightarrow} X$ onto a set $X$, the quotient quasitopology exists and has as admissible maps the $K \stackrel{\alpha}{\longrightarrow} X$ for which there exists a continuous surjective function $W \rightarrow K$ and an admissible $W \stackrel{\beta}{\longrightarrow} Y$ making the following diagram commutative.

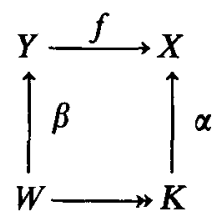

(0.9) Any continuous map $X \stackrel{f}{\longrightarrow} Y$ has a factorization as a quotient map followed by a continuous surjection and a factorization as a continuous surjection followed by an injective initial map. These factorizations are given by the image of $f$ quasitopologized as a quotient of $X$ or as a sub-space of $Y$, respectively.

(0.10) The category $\mathscr{Q}$ is cartesian closed. We will denote by $\mathscr{Q}(X, Z)$ the exponential. This means that for any pair of quasitopological spaces $X$ and $Z$, there is a quasitopological space $\mathscr{Q}(X, Z)$ such that for any other quasitopological space $Y$ there are natural bijections:

$$
\begin{array}{ll}
X \times Y \stackrel{h}{\longrightarrow} Z, & \text { continuous } \\
\hline X \stackrel{f}{\longrightarrow} \mathscr{Q}(Y, Z), & \text { continuous } \\
Y \stackrel{g}{\longrightarrow} \mathscr{Q}(X, Z), & \text { continuous }
\end{array}
$$

where $f, g$ and $h$ are related by $h(x, y)=(f(x)),(y)=(g(y))(x)$. It follows (automatically) that these bijections induce bicontinuous bijections of quasitopological spaces:

$$
\mathscr{Q}(X \times Y, Z) \approx \mathscr{Q}(X, \mathscr{Q}(Y, Z)) \approx \mathscr{Q}(Y, \mathscr{Q}(X, Z))
$$


The exponential $\mathscr{Q}(X, Z)$ is the set of continuous maps $X \longrightarrow Z$ with the quasitopology for which a map $K \stackrel{\gamma}{\longrightarrow} \mathscr{2}(X, Z)$ is admissible if and only if the corresponding $K \times X \stackrel{\eta}{\longrightarrow} Z$ is continuous (here $\eta(k, x)=(\gamma(k))(x)$ ). In view of the definition of the product $K \times X$ (see (0.4)) this amounts to saying that

$$
K \times W \stackrel{K \times \beta}{\longrightarrow} K \times X \stackrel{\eta}{\longrightarrow} Z
$$

is admissible for all admissible $W \stackrel{\beta}{\longrightarrow} X$.

(0.11) It follows from (0.10) that given $Z$ in $\mathscr{Q}$, for any quotient map $Y \stackrel{f}{\longrightarrow} X$ (respectively, initial injective $X \stackrel{i}{\rightarrow} Y$ ) the maps $\mathscr{2}(X, Z) \stackrel{\mathscr{2}(f, Z)}{\longrightarrow} \mathscr{2}(Y, Z)$ (respectively, $\mathscr{2}(Z, X) \stackrel{\mathscr{Q}(Z, i)}{\longrightarrow} \mathscr{Q}(Z, Y))$ is an initial injective. For any limit diagram $X \stackrel{\pi_{\lambda}}{\longrightarrow} X_{\lambda}$ (respectively, colimit diagram $X_{\lambda} \stackrel{g_{\lambda}}{\longrightarrow} X$ ), the diagram

$$
\mathscr{Q}(Z, X) \stackrel{\mathscr{Q}\left(Z, \pi_{\lambda}\right)}{\longrightarrow} \mathscr{Q}\left(Z, X_{\lambda}\right)
$$

(respectively, $\mathscr{Q}(X, Z) \stackrel{\mathscr{Q}\left(g_{\lambda}, Z\right)}{\longrightarrow} \mathscr{Q}\left(X_{\lambda}, Z\right)$ ) is a limit diagram.

(0.12) Given any quasitopological space $X$, the index category of $X$, denoted $\Gamma_{X}$, is the category whose objects are the admissible maps $K \stackrel{\alpha}{\longrightarrow} X$ and whose morphisms are the commutative triangles (with $f$ continuous):

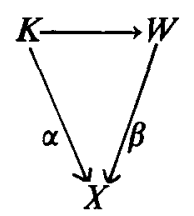

There is an obvious functor $\Gamma_{X} \longrightarrow \mathscr{Q},(K \stackrel{\alpha}{\longrightarrow} X) \mapsto K$ and it is not difficult to check that the family $K \stackrel{\alpha}{\longrightarrow} X$ ( $\alpha$ admissible) is a colimit diagram for this functor.

(0.13) If $X$ is separated (in the sense of (1.8) below), then $\Gamma_{X}$ is a filtered category (MacLane (1971)). The proof is given below in (1.13).

\section{Relationship between uniform spaces and Spanier's quasitopologies}

Each compact space $K$ will be considered as a uniform space (to be denoted also by $K$ ) by assigning to it the unique uniformity that induces the given compact topology. We recall that for any uniform space $T$, a continuous map $K \longrightarrow T$ for the uniform topology of $T$ is automatically uniformly continuous (abbreviated $u$-continuous in the sequel). 
Each uniform space $T$ determines canonically a quasitopological space by taking as admissible maps the u-continuous maps $K \longrightarrow T$.

(1.1) Definition. Given a uniform space $T$, the u-continuous functions $K \longrightarrow T$ are the admissible maps of a quasitopology (on the underlying set of $T$ ) that we will call the uniform quasitopology of $T$ and will denote by $q T$.

It is clear that if $T \stackrel{f}{\longrightarrow} S$ is u-continuous, then $q T \stackrel{f}{\longrightarrow} q S$ is a continuous map of quasitopological spaces. Thus $q$ is a functor $\mathscr{U} \stackrel{q}{\longrightarrow} \mathscr{Q}$ from the category $\mathscr{U}$ of uniform spaces and u-continuous maps into 2 .

On the other hand, each quasitopological space $X$ determines canonically a uniformity on its underlying set as the final uniformity determined by the admissible maps. More concretely:

(1.2) Definition. Given a quasitopological space $X$, the admissible-generated uniformity on $X$, denoted by $\ell X$, is defined by the following bijection:

$$
\frac{\ell X \stackrel{f}{\longrightarrow} T, \quad \text { u-continuous }}{K \stackrel{\alpha}{\longrightarrow} X \stackrel{f}{\longrightarrow} T, \quad \text { u-continuous for each admissible } \alpha}
$$

If $K$ is a compact space, we have already observed that it can be considered as a quasitopology (paragraph following (0.3)) and as a uniform space (beginning of this section). We will delete the $q$ in $q K$ and write simply $K$. Since clearly $\ell q K=K$ can be proved, we can also drop the $\ell$ and so in the sequel we write $K=q K=\ell K$.

(1.3) Proposition. Let $X$ be a quasitopological space. Then $\ell X$ is the initial uniformity determined by the family of all $X \stackrel{f}{\longrightarrow} q T$ continuous, where $T$ is an arbitrary uniform space.

Proof. Observe that by definition we have bijections:

$$
\begin{aligned}
& \ell X \stackrel{f}{\longrightarrow} T, \quad \text { u-continuous } \\
& \stackrel{\alpha}{\longrightarrow} X \stackrel{f}{\longrightarrow} T, \quad \text { u-continuous for all } \alpha \\
& K \stackrel{\alpha}{\longrightarrow} X \stackrel{f}{\longrightarrow} q T, \text { admissible for all } \alpha \\
& X \stackrel{f}{\longrightarrow} q T, \quad \text { continuous }
\end{aligned}
$$

and this shows that $\ell X$ is the initial uniformity for the family described.

Remark 1. Although it is true that the uniform topology of a uniform space is the initial topology of the family of u-continuous real-valued functions, its 
uniformity need not be the initial uniformity of this same family, even for metric spaces (they are different for the metric space: $T$ any uncountable set,

$$
d(x, y)=1 \quad \text { if } x \neq y, d(x, x)=0) \text {. }
$$

This type of problem is studied in Ginsburg and Isbell (1959). Using the fact that all uniformities are generated by metrics, we can reduce the family $X \stackrel{f}{\longrightarrow} q T$ to just metric (uniform) spaces $T$.

REMARK 2. Notice that if $\ell X \stackrel{f}{\longrightarrow} T$ is continuous, for all admissible $K \stackrel{\alpha}{\longrightarrow} X$ the composite $K \stackrel{f \circ \alpha}{\longrightarrow} T$ is continuous, hence u-continuous by compactness. But then, by Definition (1.2), $\ell X \stackrel{f}{\longrightarrow} T$ is also u-continuous. Thus, for the uniform space $\ell X$, continuous functions $\ell X \longrightarrow T$ are automatically u-continuous or, in other words, the uniformity of $\ell X$ is fine in the sense of Isbell (1964). This will be used below, in the proof of (1.9).

(1.4) Proposition. The functor $\mathscr{U} \stackrel{q}{\longrightarrow} \mathscr{Q}$ has a left adjoint $\mathscr{Q} \stackrel{\ell}{\longrightarrow} \mathscr{U}$. Furthermore, $q \ell q=q$ and $\ell q \ell=\ell$.

Proof. The first statement only means that there is a natural bijection

$$
\stackrel{\ell X \longrightarrow T, \mathrm{u} \text {-continuous }}{X \longrightarrow q T, \text { continuous }}
$$

and this is the content of the proof of (1.3). For the second part we observe that using the adjunction (a) for the identities $\ell X \longrightarrow \ell X$ and $q T \longrightarrow q T$ we get that the identity map is uniformly continuous for $\ell_{q} T \longrightarrow T$ and continuous for $X \longrightarrow \phi \ell X$. But then we get that $\ell X \longrightarrow \ell q \ell X \longrightarrow X$ is u-continuous and that $q T \longrightarrow q \ell q T \longrightarrow q T$ is continuous. This finishes the proof.

REMARK. For $X \in \mathscr{Q}$ let us denote by $t X$ the final topology for the family of admissible maps. Since $X \longrightarrow q \ell X$ is continuous, for each admissible $K \longrightarrow X$ the map $K \longrightarrow X \longrightarrow \ell X$ is u-continuous, and so $t X \longrightarrow \ell X$ is continuous. But $t X$ need not coincide with the uniform topology of $\ell X$ (when $t X$ is not completely regular, for example). In fact the following example shows that they can be very different. Let $X$ denote the quasitopology on the set $\mathbf{N}=\{1,2, \ldots\}$ of natural numbers defined by: $K \stackrel{\alpha}{\longrightarrow} X$ is admissible if $\alpha(K)$ is a finite subset of N. It is easy to see that $\ell X$ is the indiscrete uniformity (that is, the only entourage is $X \times X$ itself). However, $t X$ is the Fréchet topology on $\mathbf{N}$ (closed $=$ finite).

By standard categorical routine (MacLane (1971), v.5, Theorem 1), from Proposition (1.4), we get 
(1.5) Corollary. The functor $q: \mathscr{U} \longrightarrow \mathscr{Q}$ preserves all limits and $\ell: \mathscr{Q} \longrightarrow \mathscr{U}$ preserves all colimits. In particular: the uniform quasitopology of a product is the product quasitopology, the quasitopology of a relative uniformity is the relative quasitopology, and if $T \stackrel{f_{\alpha}}{\longrightarrow} T_{\alpha}$ is an initial family in $\mathscr{U}$, then $q T \stackrel{q f_{\alpha}}{\longrightarrow} q f_{\alpha}$ is an initial family in 2. The corresponding dual statements hold for $\ell$.

(1.6) Definition. A uniform space $T$ is uniformly compactly generated (abbreviated uc-generated) if $T=\ell q T$.

This means that the uniformity of $T$ is determined by the u-continuous maps $K \stackrel{\alpha}{\longrightarrow} T$ with compact domain $K$, in the sense that a function $T \stackrel{f}{\longrightarrow} S$ is uniformly continuous if and only if all compositions $K \stackrel{\alpha}{\longrightarrow} T \stackrel{f}{\longrightarrow} S$ are.

We remark that the uniform topology of a uc-generated space need not be a $k$-space or a compactly generated topological space (see Clark (1969), Example 2.12). In fact we have:

(1.7) Proposition. A uniform space $T$ is uc-generated if and only if $T$ is fine and the uniform topology of $T$ is a $K_{\mathbf{R}}$-space.

Before we prove (1.7) let us recall that a topological space $Z$ is a $K_{\mathbf{R}}$-space if it is completely regular and a real valued function $Z \stackrel{f}{\longrightarrow} \mathbf{R}$ is continuous if and only if the compositions $K \stackrel{\alpha}{\longrightarrow} Z \stackrel{f}{\longrightarrow} \mathbf{R}$ are continuous for all continuous $\alpha$ with compact domain $K$. We can write, for $Z$, a $K_{\mathbf{R}}$-space:

$\stackrel{Z \longrightarrow \mathbf{R}, \quad \text { continuous }}{X \stackrel{\alpha}{\longrightarrow} Z \longrightarrow \mathbf{R}, \text { continuous (for all } \alpha \text { ) }}$

Proof of (1.7). Let $T$ and $S$ be uniform spaces. Then

\begin{tabular}{ll}
$\ell q T \longrightarrow S$, & u-continuous, if and only if \\
\hline$T \longrightarrow q S$, & continuous, if and only if \\
\hline$K \stackrel{\alpha}{\longrightarrow} T \longrightarrow S$, & continuous (for all $\alpha$ ), if \\
\hline$T \longrightarrow S$, & continuous, if \\
\hline$T \longrightarrow S$, & u-continuous
\end{tabular}

If $T=\ell \phi T$, then (1) through (5) are equivalent and therefore $T$ is fine (because (4) $\Leftrightarrow(5)$; see Remark 2 to Proposition (1.3)). Also the equivalence (3) $\Leftrightarrow(4)$ for $S=\mathbf{R}$ gives that the uniform topology is a $K_{\mathbf{R}}$-space.

Consider now the case where $T$ is a $K_{\mathbf{R}}$-space. Then, using the fact that $S$ is 
completely regular we get

(4) $T \longrightarrow S$, continuous, if and only if

$$
T \longrightarrow S \stackrel{\beta}{\longrightarrow} \mathbf{R},
$$
continuous (for all $\beta$ ), if and only if

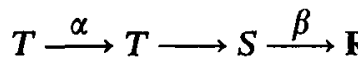

continuous (for all $\alpha$, for all $\beta$ ), if and only if

$$
K \stackrel{\alpha}{\longrightarrow} T \longrightarrow S,
$$

continuous (for all $\alpha$ )

If, further, $T$ is fine, then (4) $\Leftrightarrow(5)$ and so all (1) through (7) are equivalent. In particular (1) $\Leftrightarrow(5)$ and $T=\ell q T$. This ends the proof.

Observe that there are $K_{\mathbf{R}}$-spaces that are not compactly generated topological spaces (uncountable products of denumerable discrete spaces, for example). This is done in complete detail in Beckenstein et al. (1977), 2.2 and 2.3 and notably Example 2.3-1.

Now we turn to separation properties. The following separation axiom has been studied in the literature: a quasitopological space $X$ is separated if the image of any admissible map is closed in the admissible generated topology, that is, in $t X$ (see Remark to Proposition (1.4)). This property is closely related to a weak separation axiom for topological spaces. In fact, $X$ is separated if and only if $t X$ is weakly Hausdorff (see Clark (1969), Day (1972), Dubuc (1977), Lamartin (1977), McCord (1970)). We introduce now the analogous concept within the context of uniform spaces.

(1.8) Definition. A quasitopological space $X$ is uniformly separated (abbreviated $u$-separated) if the image of any admissible map is closed for the admissible generated uniformity. That is, for any admissible $K \stackrel{\alpha}{\longrightarrow} X$, Image $(\alpha)$ is a closed subset of $\ell X$.

Since $t X \stackrel{\text { id }}{\longrightarrow} \ell X$ is continuous (Remark after (1.4)), it follows that any useparated quasitopology is also separated. The converse, however, is false in view of the following result:

(1.9) Proposition. For a quasitopological space $X$ the following properties are equivalent:

(a) continuous functions $X \stackrel{f}{\longrightarrow}[0,1]$ into the unit real interval $[0,1]$ separate disjoint admissible maps. That is, given admissible map $K \stackrel{\alpha}{\longrightarrow} X$ and $W \stackrel{\beta}{\longrightarrow} X$ with $\{(k, w) ; \alpha(k)=\beta(w)\}=\varnothing$ there exists a continuous function $X \stackrel{f}{\longrightarrow}[0, \mathrm{f}]$ such that $f \circ \alpha=0$ and $f \circ \beta=1$; 
(b) continuous functions $X \stackrel{f}{\longrightarrow}[0,1]$ separate points;

(c) the uniform space $\ell X$ is Hausdorff;

(d) $X$ is u-separated.

Proof. (a) $\Rightarrow(b)$ is immediate since every point is the image of an admissible map according to $(\mathrm{Q} 1)$ in (0.2).

(b) $\Rightarrow$ (c) is also immediate since $X \stackrel{f}{\longrightarrow}[0,1]$ is continuous if and only if $\ell X \stackrel{f}{\longrightarrow}[0,1]$ is u-continuous.

(c) $\Rightarrow(d)$ follows since images of compact spaces in Hausdorff spaces are (compact, hence) closed.

(d) $\Rightarrow(\mathrm{a})$ : the two images $\alpha(K)$ and $\beta(W)$ are closed disjoint sets with the Heine-Borel covering property in the completely regular space $\ell X$. Then there exists $\ell X \stackrel{f}{\longrightarrow}[0,1]$ continuous, $f=0$ on $\alpha(K)$ and $f=1$ on $\beta(W)$ (see Kelley (1955)). According to Remark 2 to Proposition $(1.3), \ell X \stackrel{f}{\rightarrow}[0,1]$ is ucontinuous, hence $X \stackrel{f}{\longrightarrow}[0,1]$ is continuous and (a) follows.

(1.10) Corollary. (a) Products of u-separated quasitopologies are u-separated. (b) If $X$ is u-separated and $Y \stackrel{i}{\longrightarrow} X$ is a continuous injective function, then $Y$ is $u$-separated. (c) For any quasitopological space $X, X$ is $u$-separated if and only if the exponential $\mathfrak{Z}(Y, X)$ is $u$-separated for all $Y$.

Proof. From part (b) in (1.9) we get easily (a) and (b) in (1.10). Now, since $\mathscr{Z}$ is cartesian closed (see (0.10)) it follows that for each $y \in Y$, the evaluation $\operatorname{ev}_{y}(f)=f(y)$ is continuous $\mathscr{2}(Y, X) \stackrel{\mathrm{ev}_{y}}{\longrightarrow} X$ and therefore there is a continuous injective of $\mathscr{Q}(Y, X)$ into a product $\prod X$. Thus if $X$ is u-separated and then so is $\mathscr{2}(Y, X)$, from (a) and (b) above. For the converse, just take $Y=1$ and use $\mathscr{2}(1, X) \approx X$.

(1.11) COROLlaRY. For any Hausdorff uniform space $T$ the uniform quasitopology $q T$ is u-separated.

Proof. Notice that the identity $\ell_{q} T \longrightarrow T$ is u-continuous (proof of (1.4)) and therefore $\ell q T$ is also Hausdorff. Now use (c) $\Rightarrow$ (d) in (1.9) to conclude that $q T$ is u-separated.

Given a compact subset $K$ of a $T_{1}$ completely regular topological space, any continuous real (or complex) valued function defined on $K$ admits a continuous extension to the whole space (see Kelley (1955)). This property carries over to u-separated quasitopologies. More precisely: 
(1.12) Proposition. Let $F$ denote the real field or the complex field. A quasitopological space $X$ is u-separated if and only if every injective admissible $K \stackrel{\alpha}{\longrightarrow} X$ has the F-valued extension property, that is, given any continuous function $K \stackrel{g}{\longrightarrow} F$ there exists a continuous function $X \stackrel{f}{\longrightarrow} q F$ such that $f \circ \alpha=g$.

Proof. Suppose $X$ is u-separated. Then the image of $K \stackrel{\alpha}{\longrightarrow} X$ is a compact subset of $\ell X$, and $K \stackrel{\alpha}{\longrightarrow} X$ is a compact subset of $\ell X$, and $K \stackrel{\alpha}{\longrightarrow} \alpha(K)$ is a homeomorphism. Then, as remarked above, $\alpha(K) \stackrel{\alpha^{-1}}{\longrightarrow} K \stackrel{g}{\longrightarrow} F$ has a continuous extension $\ell X \stackrel{f}{\longrightarrow} F$ which being automatically u-continuous, defines a continuous $X \longrightarrow q F$ that satisfies $f \circ \alpha=g$ by definition. Suppose now that every injective admissible has the $F$-extension property and consider, for $x \neq y$ in $X$, the inclusion $\{x\} \cup\{y\} \stackrel{i}{\longrightarrow} K$. From (Q1) and (Q3) in (0.2), it follows that $i$ is admissible, and extending $g(x)=0, g(y)=1$ we get a continuous $X \stackrel{f}{\longrightarrow} F$ that separates $x$ and $y$ and that we may assume also satisfies $0 \leqslant f \leqslant 1$ after replacing $f$ by $\inf (1, \max (0, f))$. Then (b) $\Rightarrow(\mathrm{d})$ in (1.9) applies to give that $X$ is u-separated.

Now we prove the statement $(0.13)$. More precisely:

(1.13) Proposition. Let $X$ be a quasitopological space. If $X$ is u-separated, then its index category $\Gamma_{X}$ (see (0.12)) is filtered, and the maps $K \stackrel{\alpha}{\longrightarrow} X$ where $\alpha$ is an injective admissible map with the F-valued extension property, form a filtered colimit diagram in 2 . Conversely, if there is such a filtered colimit diagram, then $X$ is u-separated.

Proof. Suppose $X$ is u-separated, and let $K \stackrel{\alpha}{\longrightarrow} X$ and $W \stackrel{\beta}{\longrightarrow} X$ be admissible maps. Since $K \stackrel{\alpha}{\longrightarrow} \ell X$ and $W \stackrel{\beta}{\longrightarrow} \ell X$ are continuous and $\ell X$ is Hausdorff ((c) in (1.19)), then the pull back

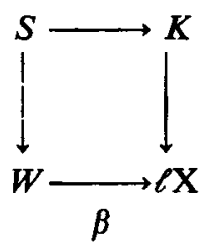

is compact $(S=\{(k, w) ; \alpha(k)=\beta(W)\} \subset K \times W$ is closed in $K \times W$ because $\ell X$ is Hausdorff), and then $\Gamma_{X}$ is filtered by definition (see McLane (1971), Chapter IX). Let us now observe that for any $K \stackrel{\alpha}{\longrightarrow} X$ in $\Gamma_{X}$, the image $W=\alpha(K)$ is compact (in the relative uniformity of $\ell X$ ) and the identity $W \stackrel{i}{\longrightarrow} X$ is admissible by (Q3) in (0.2). This, together with (1.12) shows that the injective admissible maps with 
the $F$-valued extension property form a cofinal sub-category of $\Gamma_{X}$ and, therefore, from the first part of the proof it follows that it is itself filtered, as claimed.

Suppose now that $K \stackrel{\alpha}{\longrightarrow} X$ is a filtered colimit diagram (with $\alpha$ admissible having the $F$-valued extension property). By definition (see (0.6)) and since it is a filtered diagram, for each admissible $W \stackrel{\beta}{\longrightarrow} X$ there is an $\alpha$ surjective $D \stackrel{f}{\longrightarrow} W$ with

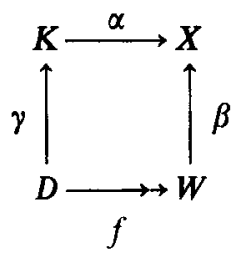

commutative. But then there is a factorization

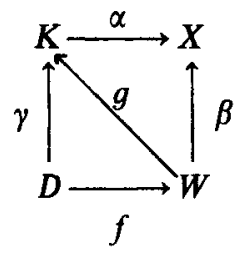

with $g$ continuous (by compactness of $D$ and $\dot{W}$ we have

$$
\left.g^{-1}(\text { closed })=f\left(\gamma^{-1}(\text { closed })\right)=f(\text { compact })=\text { compact }=\text { closed }\right) .
$$

Since $\alpha$ has the $F$-valued extension property and $\beta(W) \subset \alpha(K)$, we see that continuous functions $X \longrightarrow F$ separate points of $\beta(W)$. But any pair of points of $X$ belong to some $\beta(W)$ (by $(\mathrm{Q} 3)$ ), we get (b) in (1.9) and therefore $X$ is u-separated.

\section{The enrichment of the category of uniform spaces over the category of quasitopologies}

Since 2 is a cartesian closed category we can consider the problem of whether the situation described in Proposition (1.4) has an enrichment in such a way that the enriched 2-valued contravariant representable functors have enriched left adjoints. This is actually the case, and we proceed to show how it is done.

First, we recall some needed properties of uniform spaces. Given $S$ and $T$ uniform spaces, we denote with $F(S, T)$ the set of all functions from $S$ to $T$, and with $\mathscr{U}_{u}(S, T)$ (respectively, $\mathscr{U}_{\pi}(S, T)$ ) the uniform space of all u-continuous functions from $S$ to $T$ with the uniformity of the uniform convergence on $S$ (respectively, the pointwise convergence on $S$ ). 
(2.1) LEMMA. Let $R, S, T$ be uniform spaces and let $R \times S \stackrel{h}{\longrightarrow} T, R \stackrel{f}{\longrightarrow} F(S, T)$ and $S \stackrel{g}{\longrightarrow} F(R, T)$ be any three functions related by the formulas

Then:

$$
h(r, s)=g(s)(r)=f(r)(s) .
$$

(a) there is a bijection between the $u$-continuous $g$ with values in $\mathscr{U}_{u}(R, T)$ and the $u$-continuous $f$ with values in $\mathscr{U}_{\pi}(S, T)$ and such that $\operatorname{Image}(f)$ is a uniformly equicontinuous set. In symbols:

$S \longrightarrow \mathscr{U}_{u}(R, T), \quad$ u-continuous

$R \longrightarrow \mathscr{U}_{\pi}(S, T), \quad$ u-continuous and Image $(f)$ a uniformly equicontinuous set.

(b) given $J \subset \mathscr{U}_{\pi}(R, S)$ and $H \subset \mathscr{U}_{\pi}(S, T)$ any two uniformly equicontinuous sets with the induced uniformity (that is, the uniformity of pointwise convergence), then the composition function

$$
J \times H \longrightarrow \mathscr{U}_{\pi}(R, T)
$$

is continuous and its image is a uniformly equicontinuous set;

(c) given any compact space $K=R$, there is a bijection

$$
\frac{K \times S \longrightarrow T, \text { u-continuous }}{K \stackrel{f}{\longrightarrow} \mathscr{U}_{u}(S, T), \text { u-continuous; }}
$$

(d) the bijection given by (c) is an isomorphism of uniform spaces:

$$
\mathscr{U}_{u}(K \times S, T)=\mathscr{U}_{u}\left(K, \mathscr{U}_{u}(S, T)\right)
$$

(e) if $T \stackrel{f_{\alpha}}{\longrightarrow} T_{\alpha}$ is a limit diagram of uniform spaces (in particular, the uniformity of $T$ is the smallest that makes all $f_{x} u$-continuous) and $S$ is any uniform space, then the diagram

$$
\mathscr{U}_{u}(S, T) \stackrel{\mathscr{U}_{u}\left(S, f_{\alpha}\right)}{\longrightarrow} \mathscr{U}_{u}\left(S, T_{\alpha}\right)
$$

is also a limit diagram of uniform spaces, where

$$
\mathcal{U}_{u}\left(S, f_{\alpha}\right)(f)=f_{\alpha} \circ f \text { for } S \stackrel{f}{\longrightarrow} T \text {. }
$$

Proof. (a) Follows from Bourbaki (1940), Chapter 10, Section 2, Proposition 1 (with $R, S, T$ taking the place of $T, X, Y$ there); (b) follows from loc. cit., Chapter 10, Section 2, Corollary 5 of Proposition 1 and Exercise 4,6; (c) follows from (a) above and loc cit., Chapter 10, Section 2, Proposition 2 and Theorem 2 (necessary condition (a)) and loc. cit., Chapter 2, Section 4, Proposition 2; (d) follows from loc. cit., Chapter 10, Section 1, Proposition 2; and finally (e) follows from loc. cit., Chapter 10, Section 1, Propositions 3 and 4. 
Observe that the conclusion in part (b) cannot be strengthened to 'u-continuous' instead of just 'continuous'. In fact, if $R=T=S=$ real line with its standard uniformity and $J=H=\{f: \mathbf{R} \longrightarrow \mathbf{R} ;|f(x)-f(y)| \leqslant|x-y|$ for all $x, y \in \mathbf{R}\}$, then $J \times H \longrightarrow \mathscr{U}_{u}(\mathbf{R}, \mathbf{R}),(f, g) \longmapsto g \circ f$ is not u-continuous. To show that, let $x_{1}, x_{2}, \ldots, x_{n}$ be arbitrary and $\delta>0$, and set $L=\sup \left\{\left|g_{1}\left(f_{1}(0)\right)-g_{2}\left(f_{2}(0)\right)\right|\right\}$, the supremum taken over all $\left(f_{1}, f_{2}\right),\left(g_{1}, g_{2}\right)$ with

$$
\left|f_{1}\left(x_{k}\right)-f_{2}\left(x_{k}\right)\right|<\delta \text { and }\left|g_{1}\left(x_{k}\right)-g_{2}\left(x_{k}\right)\right|<\delta \quad \text { for } k=1,2, \ldots, n \text {. }
$$

It suffices to show that $L=\infty$. But the supremum can only decrease when we add more $x_{k}$ 's, so that we may always assume that $x_{n}=0$. Then by definition,

$$
\begin{aligned}
&\left|g_{1}\left(f_{1}(0)\right)-g_{2}\left(f_{2}(0)\right)\right| \geqslant\left|g_{1}\left(f_{1}(0)\right)-g_{2}\left(f_{1}(0)\right)\right|-\left|g_{2}\left(f_{1}(0)\right)-g_{2}\left(f_{2}(0)\right)\right| \\
&\left.\geqq\left|g_{1}\left(f_{1}(0)\right)-q_{2}\left(f_{1}(0)\right)\right|\right\}-\delta,
\end{aligned}
$$

and so $L \geqq \sup \left\{g_{1}\left(f_{1}(0)\right)-g_{2}\left(f_{1}(0)\right) \mid\right\}-\delta$. But $f_{1}(0)$ ranges over all of $\mathbf{R}$, and so the last supremum is obviously infinite.

Suppose now that $S$ and $T$ are given uniform spaces, and let $M$ be the set of u-continuous functions from $S$ to $T$. The uniformity equicontinuous subsets of $M$ from an ideal $I$ in the Boolean algebra of all subsets of $M$, and all singletons belong to $I$. This shows that the family of all maps $K \stackrel{\alpha}{\longrightarrow} M$, where $K$ is compact and $\alpha(K) \in I$, defines a quasitopology on $M$, that we will denote by $\mathscr{U}_{I}(S, T)$.

(2.2) Definition. Given any uniform spaces $S$ and $T$, we denote by $\mathscr{U}(S, T)$ the set of $u$-continuous functions from $S$ to $T$ endowed with the quasitopology supremum of $\mathscr{U}_{I}(S, T)$ and $q \mathscr{U}_{\pi}(S, T)$. More precisely, $K \stackrel{\alpha}{\longrightarrow} \mathscr{U}(S, T)$ is admissible if and only if

(a) $\alpha(K)$ is a uniformly equicontinuous set, and

(b) $K \stackrel{\alpha}{\longrightarrow} \mathscr{U}_{\pi}(S, T)$ is u-continuous.

This definition is chosen so that, according to Lemma (2.1)(a), a map $K \stackrel{\alpha}{\longrightarrow} \mathscr{U}(S, T)$ is admissible if and only if $S \longrightarrow \mathscr{U}_{u}(K, T)$ is u-continuous.

Now we come to the result announced at the beginning of the section.

(2.3) Proposition. The category $\mathscr{U}$ has a structure of 2-category in such a way that $q$ and $\ell$ become 2-adjoint 2-functors.

Before we prove (2.3) we will explain its parts:

(i) $\mathscr{U}$ is a 2 -category. We must prove that for any three uniform spaces $R, S$ and $T$ the composition of functions

$$
\mathscr{U}(R, S) \times \mathscr{U}(S, T) \longrightarrow \mathscr{U}(R, T)
$$

is a continuous map of quasitopologies. 
(ii) $q$ and $\ell$ are 2 -functors. This means that the inclusion maps

$$
\mathscr{U}(S, T) \stackrel{q}{\longrightarrow} \mathscr{2}(q S, q T) \text { and } \mathscr{2}(X, Y) \stackrel{\ell}{\longrightarrow} \mathscr{U}(\ell X, \ell Y)
$$

are continuous.

(iii) $q$ and $\ell$ are 2 -adjoints. This means that the bijection (a) in the proof of Proposition (1.4) is an ismorphism of quasitopologies, that is $\mathscr{U}(\ell X, T)=\mathscr{2}(X, q T)$ as quasitopological spaces.

Proof. (i) follows from (0.4) and Lemma (2.1)(b).

Next we will prove (iii) by showing that both quasitopologies have exactly the same admissible maps. Consider the following chain of bijections (or logical equivalences):

$$
\begin{array}{rll}
\text { (1) } & K \longrightarrow 2(X, q T), & \text { admissible } \\
\text { 2) } & K \times X \longrightarrow q T, & \text { continuous } \\
\text { (3) } & \ell(K \times K) \longrightarrow T, & \text { u-continuous } \\
\text { (4) } & K \times W \stackrel{\text { id } \times \beta}{\longrightarrow} K \times K \longrightarrow T, & \text { u-continuous (for all } W \stackrel{\beta}{\longrightarrow} X) \text { admissible } \\
\text { (5) } & W \stackrel{\beta}{\longrightarrow} \ell X \longrightarrow \mathscr{U}_{u}(K, T), & \text { u-continuous (for all } W \stackrel{\beta}{\longrightarrow} X \text { admissible) } \\
\text { (6) } & K \longrightarrow \mathscr{U} \longrightarrow \mathscr{U}_{u}(K, T), & \text { u-continuous }
\end{array}
$$

They hold for the following reasons: (1) by the cartesian closed structure of $\mathscr{Q}$ (0.10) (above); (2) from Proposition (1.4); (3) from (0.4) and (1.2); (4) from (2.1)(c); (5) again from Definition (1.2) and finally (6) from (2.1)(a) and Definition (2.2).

Now we observe that (ii) follows from (i) and (iii) since any pair of adjoint functors between 2 -categories which are 2 -adjoint are automatically 2 -functors. In this case we can get this result as follows:

and

$$
\mathscr{U}(S, T) \stackrel{\mathscr{U}(\mathrm{id}, T)}{\longrightarrow} \mathscr{U}(\ell \phi S, T)=\mathscr{Q}(q S, q T)
$$

$$
\mathscr{2}(X, T) \stackrel{\mathscr{Q}(X, \mathrm{id})}{\longrightarrow} \mathscr{Q}(X, q \ell Y)=\mathscr{U}(\ell X, \ell Y) .
$$

The two arrows on the left are continuous since (as observed in the proof of (1.4)) $\ell q S \stackrel{\text { id }}{\longrightarrow} S$ and $Y \stackrel{\text { id }}{\longrightarrow} q \ell Y$ are, respectively, u-continuous and continuous. This completes the proof of (2.3).

REMARK 1. Notice that since the inclusion $\mathscr{U}(S, T) \hookrightarrow \mathscr{Q}(q S, q T)$ is continuous, if follows that the quasitopology $\mathscr{U}(S, T)$ is u-separated when $T$ is a Hausdorff uniform space (use (1.9)(c), and (1.10)). 
REMARK 2. Notice that a function $K \longrightarrow \mathscr{2}(\not{q} S, q T)$ is admissible if and only if the corresponding functions $\ell_{q} S \longrightarrow \mathscr{U}_{u}(K, T)$ is u-continuous. To see this make $X=q S$ in the proof of (iii) above. In symbols:

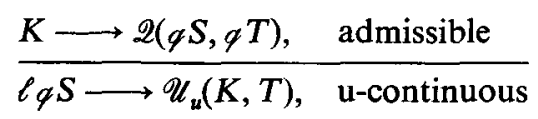

Clearly

$$
\begin{array}{ll}
K \longrightarrow \mathscr{U}(S, T), & \text { admissible } \\
S \longrightarrow \mathscr{U}_{u}(K, T), & \text { u-continuous }
\end{array}
$$

by Definition (2.2) and (2.1)(a).

On the other hand, we have, by u-continuity of $\ell q S \longrightarrow S$, that

$$
\mathscr{U}(S, T) \stackrel{\mathscr{U}(\mathrm{id}, T)}{\longrightarrow} \mathscr{U}(\ell q S, T)=\mathscr{Q}(q S, q T) .
$$

We see then that: if $S$ is uc-generated (Definition (1.6)) then $\mathscr{U}(S, T)$ has the initial quasitopology of $\mathscr{U}(S, T) \longrightarrow \mathscr{2}(q S, q T)$. Using (*) and (**) we can see that it is only in this case. In fact, if for all $T$ the admissible maps for $\mathscr{U}(S, T)$ are exactly those of $\mathscr{Q}(\not S, q T)$, then there is a correspondence (by $\left({ }^{*}\right)$ and $\left.\left({ }^{*}\right)\right)$ :

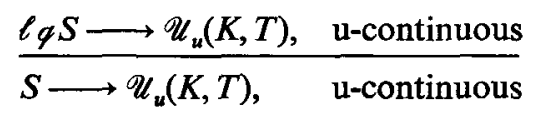

and so taking $K=1$ we conclude that $S=\ell q S$.

REMARK 3. If $T \stackrel{f_{\alpha}}{\longrightarrow} T_{\alpha}$ is a limit diagram of uniform spaces and $S$ is a uniform space, the diagram $\mathscr{U}(S, T) \stackrel{\mathscr{U}\left(S, f_{\alpha}\right)}{\longrightarrow} \mathscr{U}\left(S, T_{\alpha}\right)$ is a limit diagram of quasitopologies. In fact the composition $K \stackrel{\beta}{\longrightarrow} \mathfrak{U}(S, T) \longrightarrow \mathscr{U}\left(S, T_{\alpha}\right)$ is admissible if and only if $S \longrightarrow \mathscr{U}_{u}(K, T) \longrightarrow \mathscr{U}_{u}\left(K, T_{a}\right)$ is u-continuous. Using (2.1)(e) we conclude that if this happens for all $\alpha$, then $S \longrightarrow \mathscr{U}_{u}(K, T)$ is u-continuous; so $K \stackrel{\beta}{\longrightarrow} \mathscr{U}(S, T)$ is admissible.

For $T$ a uniform space and $X$ a quasitopology we introduce now a uniformity on the set of continuous maps $X \longrightarrow q T$. More precisely:

(2.4) Definition. For $X$ in $\mathscr{Q}$ and $T$ in $\mathscr{U}$ the uniform space $\mathscr{C}(X, T)$ is the set of continuous functions $X \longrightarrow q T$ provided with the uniformity of uniform convergence on admissible maps, defined as the initial uniformity determined by the functions

$$
\mathscr{C}(X, T) \stackrel{\mathscr{C}(\alpha, T)}{\longrightarrow} \mathscr{U}_{u}(K, T) ; \quad \mathscr{C}(\alpha, T)(f)=f \circ \alpha,
$$

where $K \stackrel{\alpha}{\longrightarrow} X$ ranges over all the admissible maps. 
Observe that $\mathscr{C}(K, T)=\mathscr{U}_{u}(K, T)$ for any compact $K$.

REMARK 1. Given a uniform space $T$ and a continuous function $K \stackrel{f}{\longrightarrow} W$ of compact spaces, it is easy to check that the induced function

$$
\mathscr{C}(W, T) \stackrel{\mathscr{C}(f, T)}{\longrightarrow} \mathscr{C}(K, T)
$$

is uniformly continuous. Then for any quasitopological space $X, \mathscr{C}(-, T)$ is a functor from the index category $\Gamma_{X}$ (see $\left.(0.12)\right)$ to $\mathscr{U}$; and it follows easily that the diagram $\mathscr{C}(X, T) \stackrel{\mathscr{C}(\alpha, T)}{\longrightarrow} \mathscr{C}(K, T)$ is a limit diagram.

REMARK 2. Notice that since all maps $1 \longrightarrow X$ are admissible (here 1 is compact space with one point) it follows that for each $x \in X$ the evaluation $\mathscr{C}(X, T) \stackrel{\mathrm{ev}_{x}}{\longrightarrow} T$ at $x$ is u-continuous. Hence the uniformity of $\mathscr{C}(X, T)$ is finer than the uniformity of point-wise convergence. It follows that $\mathscr{C}(X, T)$ is Hausdorff when $T$ is Hausdorff.

RemarK 3. $\mathscr{C}(X, T)$ is complete when $T$ is complete. This can be seen by adapting the proof of Corollary 2 to Theorem 2 of Section 1 in Bourbaki (1940), Chapter 10.

The uniformities $\mathscr{C}(X, T)$ provide adjoints for the representable functors into $\mathscr{Q}$. More precisely:

(2.5) THEOREM. The category is a cotensored 2-category.

This statement just means that all the representable functors $\mathscr{U}^{\mathrm{op}} \stackrel{\mathscr{U}(-, T)}{\longrightarrow} \mathscr{Q}$ have a 2 -left adjoint. In order to prove (2.5) it will suffice to show that given any uniform space $T$ and a quasitopological space $X$, the cotensor of $T$ with $X$ exists or, in other words, that there is a uniform space $V=\mathscr{C}(X, T)$ such that we have a natural bicontinuous bijection

$$
\mathscr{U}(S, \mathscr{C}(X, T)) \cong \mathscr{2}(X, \mathscr{U}(S, T))
$$

for all $S$. We will prove that $V=\mathscr{C}(X, T)$ as defined above in (2.4) has this property.

ProOF of (2.5). Let $K$ and $W$ be compact spaces, and consider the following chain of bijections (or logical equivalences):

\begin{tabular}{ll}
$W \longrightarrow \mathscr{Q}(K, \mathscr{U}(S, T))$, & admissible \\
\hline$W \times K \longrightarrow \mathscr{U}(S, T)$, & admissible \\
\hline$S \longrightarrow \mathscr{U}_{u}(W \times K, T)$, & u-continuous \\
\hline$S \longrightarrow \mathscr{U}_{u}\left(W, \mathscr{U}_{u}(K, T)\right)$, & u-continuous \\
\hline$W \longrightarrow \mathscr{U}\left(S, \mathscr{U}_{u}(K, T)\right)$, & admissible
\end{tabular}


Here (1) follows by the cartesian closed structure of $\mathscr{Q}$ (see (0.10)), (2) and (4) by Definition (2.2) and (3) by (2.1)(d).

Since by definition $\mathscr{C}(K, T)=\mathscr{U}_{u}(K, T)$ we have just proved that

$$
\mathscr{U}(S, \mathscr{C}(K, T)) \cong \mathscr{Q}(K, \mathscr{U}(S, T))
$$

for $K$ compact and $T$ and $S$ in $\mathscr{U}$. Let now $X$ be a quasitopological space with admissible maps $K \stackrel{\alpha}{\longrightarrow} X$ and consider the families of functions:

$$
\begin{aligned}
& \mathscr{Q}(X, \mathscr{U}(S, T)) \stackrel{\mathscr{Q}(\alpha, \mathscr{U}(S, T))}{\longrightarrow} \mathscr{Q}(K, \mathscr{U}(S, T)), \\
& \mathscr{U}(S, \mathscr{C}(X, T)) \stackrel{\mathscr{U}(S, \mathscr{C}(\alpha, T))}{\longrightarrow} \mathscr{U}(S, \mathscr{C}(K, T)) .
\end{aligned}
$$

Since $K \stackrel{\alpha}{\longrightarrow} X$ is a colimit diagram (0.12) and $\mathscr{Q}$ is cartesian closed, it follows that (ii) is a limit diagram in 2 . On the other hand, from Remark 3 to Proposition (2.3) it follows that (iii) is also a limit diagram in 2 (see also Remark 1 to Definition (2.4)). The theorem follows now from (i) and the fact that the categories of indices in (ii) and (iii) coincide.

This theorem gives a solution to a problem posed in Isbell (1964), Problem A, p. 54.

Remark 1. According to (2.5), $\mathscr{U}(S, \mathscr{C}(X, T))$ and $\mathscr{2}(X, \mathscr{U}(S, T))$ have the same admissible maps from $K=1$. Therefore there is a bijection:

$$
\stackrel{S \stackrel{g}{\longrightarrow} \mathscr{C}(X, T), \quad \text { u-continuous }}{\longrightarrow} \mathscr{U}(S, T), \text { continuous }
$$

given by the formula $(g(s))(x)=(f(x))(s)$. Also, since the functor $\mathscr{U} \stackrel{\mathscr{q}}{\longrightarrow} \mathscr{Q}$ has a $\mathcal{Q}$-left adjoint $\ell$, it preserves cotensors. This means that for any quasitopological space $X$ and any uniform space $T$, the identity map $q \mathscr{C}(X, T)=\mathscr{2}(X, q T)$ is bicontinuous.

REMARK 2. Given any uniform space $T$ it follows from the 2 -adjunction between $\mathscr{C}(-, T)$ and $\mathscr{U}(-, T)$ that these functors have the following continuity property: if $X_{\lambda} \stackrel{f_{\lambda}}{\longrightarrow} X$ is any colimit diagram of quasitopological spaces, the diagram $\mathscr{C}(X, T) \stackrel{\mathscr{C}\left(f_{\lambda}, T\right)}{\longrightarrow} \mathscr{C}\left(X_{\lambda}, T\right)$ (respectively $\mathscr{U}(X, T) \stackrel{\mathscr{U}\left(f_{\lambda}, T\right)}{\longrightarrow} \mathscr{U}\left(X_{\lambda}, T\right)$ ) is a limit diagram of uniform spaces (respectively, of quasitopologies).

\section{Liftings of the enrichment}

Suppose $\mathscr{A}$ is any class of uniform spaces together with a class of $\mathscr{U}$-continuous functions between them containing all the identities and closed under compositions 
(that is, $\mathscr{A}$ is a subcategory of $\mathscr{U}$ ). Then $\mathscr{A}$ can be (automatically and canonically) endowed with a structure of 2 -category. This is simply the enriched version of the classical approach of considering $\mathscr{A}$ as a category based on sets. Furthermore, if for a given $A$ in $\mathscr{A}$ and $X$ in $\mathscr{Q}$, the cotensor (computed in $\mathscr{U}$ ) $\mathscr{C}(X, A)$ happens to belong to $\mathscr{A}$, then it will be, under the appropriate natural conditions, a cotensor in $\mathscr{A}$. More generally, let $\mathscr{A}$ be a category provided with a faithful forgetful functor $\mathscr{A} \stackrel{\|}{\longrightarrow} \mathscr{U}$ (that is, any morphism $A \longrightarrow B$ in $\mathscr{A}$ as a ucontinuous function $|A| \longrightarrow|B|)$. Then:

(3.1) Proposition. $\mathscr{A}$ has a canonical structure of 2-category in such a way that $\mathscr{A} \stackrel{\|}{\longrightarrow} \mathscr{U}$ becomes a 2 -functor and for all $A, B$ in $\mathscr{A}$, the inclusion

is an initial map.

$$
\mathscr{A}(A, B) \longrightarrow \mathscr{U}(|A|,|B|)
$$

Proof. Just define the quasitopology of $\mathscr{A}(A, B)$ to be the quasitopology induced by $\mathscr{U}(|A|,|B|)$ and the forgetful functor $\mathscr{A}(A, B) \longrightarrow \mathscr{U}(|A|,|B|)$. All properties follow easily (in particular that composition $\mathscr{A}(A, B) \times \mathscr{A}(B, C) \longrightarrow \mathscr{A}(A, C)$ is continuous).

(3.2) Definition. Given $B$ in $\mathscr{A}$ and $X$ in $\mathscr{Q}$, we will say that the cotensor $\mathscr{C}(X,|B|)$ computed in $\mathscr{U}$ 'lives' in $\mathscr{A}$ if there is an object $\mathscr{C}(X, B)$ in $\mathscr{A}$ such that

(i) $|\mathscr{C}(X, B)|=\mathscr{C}(X,|B|)$ and

(ii) given any $|A| \stackrel{\varphi}{\longrightarrow}|\mathscr{C}(X, B)|$ in $\mathscr{U}$, then $\varphi$ is a morphism in $\mathscr{A}$ if and only if for each $x \in X$ the map $|A| \stackrel{f(x)}{\longrightarrow}|B|$ is a morphism in $\mathscr{A}$ (where $f(x)(a)=\varphi(a)(x))$.

Notice that (ii) is equivalent to the fact that the family of evaluations in $x \in X$ is a | |-initial family; that is:

(ii') For each $x$ in $X$, the evaluation in $x,|\mathscr{C}(X, B)| \stackrel{\mathrm{ev}_{x}}{\longrightarrow}|B|$ is actually a morphism $\mathscr{C}(X, B) \stackrel{\mathrm{ev}_{x}}{\longrightarrow} B$ in $\mathscr{A}$, and given any $|A| \stackrel{\varphi}{\longrightarrow}|\mathscr{C}(X, B)|$ in $\mathscr{U}$, $\varphi$ is a morphism $A \stackrel{\varphi}{\longrightarrow} \mathscr{C}(X, B)$ in $\mathscr{A}$ if for all $x$ in $X$ the composite $|A| \stackrel{\mathrm{ev}_{x} \circ \varphi}{\longrightarrow}|B|$ is a morphism in $\mathscr{A}$.

(3.3) Proposition. Given $B$ in $\mathscr{A}$ and $X$ in 2 , if the cotensor $\mathscr{C}(X, B)$ computed in $\mathscr{U}$ lives in $\mathscr{A}$, then the cotensor $\mathscr{C}(X, B)$ of $X$ with $B$ exists for the canonical 2-structure on $\mathscr{A}$, and it is preserved by the forgetful $\mathcal{Q}$-functor $\mathscr{A} \stackrel{\amalg}{\longrightarrow}$.

This means that for all $A$ in $\mathscr{A}$ the formula $(\varphi(a))(x)=(f(x))(a)$ defines a bijection

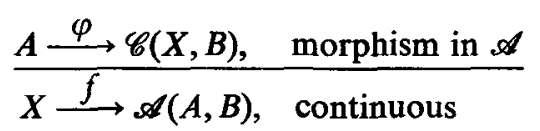


which induces a bicontinuous bijection

$$
\mathscr{A}(A, \mathscr{C}(X, B)) \approx \mathscr{2}(X, \mathscr{A}(A, B)) .
$$

Moreover, $|\mathscr{C}(X, B)|=\mathscr{C}(X,|B|)$.

Proof. We show that the object $\mathscr{C}(X, B)$ whose existence is guaranteed by (3.2) has the properties that make it the cotensor. In fact, consider

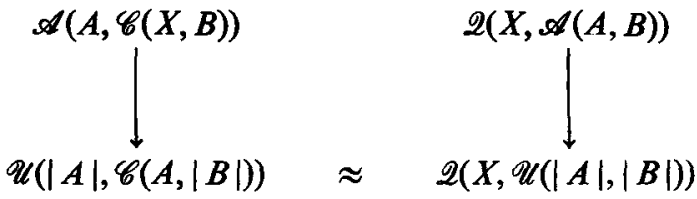

where the isomorphism in the lower level is given by (2.5). Since both vertical maps are initial maps, the proof will follow if we show that the isomorphism restricts to the upper level. But this is precisely what (3.2)(ii) says, and thus (3.3) is proved.

(3.4) CoRollary. If for all $B$ in $\mathscr{A}$ and $X$ in $\mathscr{Q}$ the cotensor $\mathscr{C}(X,|B|)$ computed in $\mathscr{U}$ lives in $\mathscr{A}$, then the canonical $\mathscr{Q}$-structure on $\mathscr{A}$ is cotensored and the $\mathscr{Q}$-functor $\mathscr{A} \stackrel{11}{\longrightarrow} \mathscr{U}$ preserves cotensors.

The conditions in Definition (3.2) are usually trivial to check in practice, where complicated arguments to prove continuity require most of the work. In this approach, continuity is guaranteed once and for all by (2.5).

Since a topological algebraic structure usually induces a uniformity on its underlying topological space in such a way that continuous algebraic morphisms become u-continuous, all standard classes of algebraic structures are examples of categories $\mathscr{A} \stackrel{\|}{\longrightarrow} \mathscr{U}$ to which Proposition (3.3) or Corollary (3.4) could be applied.

Examples are: topological groups and all their usual subcategories (Hausdorff, abelian, and so on), topological vector spaces and their subcategories (locally convex, Banach spaces, and so on), topological algebras (locally convex algebras, Banach algebras, $C^{*}$-algebras, and so on).

Since the pointwise operations on sets of structure-valued functions usually define structures belonging to the same class, Proposition (3.3) and Corollary (3.4) can be applied in most cases. For some, like Banach spaces and algebras, $C^{*}$ algebras, and so on, cotensors will not exist in general, but Proposition (3.3) can be used, notably for the case where $X$ is compact. For metrizable structures (like Fréchet spaces and algebras) a smallness condition (weaker than compactness) suffices: $X$ should be a countable quasitopology (in the following sense: $X$ is countable if there exists a sequence of admissible maps $K_{n} \stackrel{\alpha_{n}}{\longrightarrow} X$ such that every admissible $K \stackrel{\alpha}{\longrightarrow} X$ factors through some $\alpha_{n}$ as $\alpha=\alpha_{n} \circ f$ with $K \stackrel{f}{\longrightarrow} K_{n}$ 
continuous. (This amounts to saying that the index category $\Gamma_{X}$ has a countable cofinal subcategory.) Countable quasitopologies correspond to hemi-compact spaces in the sense of Arens (1946) or Michael (1952), p. 22.

A different type of situation to which Proposition (3.3) also applies arises when the functor $\mathscr{A} \stackrel{\|}{\longrightarrow} \mathscr{U}$ is full, that is, when all u-continuous functions $|A| \longrightarrow|B|$ are morphisms in $\mathscr{A}$. In this case if the cotensor $\mathscr{C}(X, B)$ belongs to $\mathscr{A}$, the condition (ii) in Definition (3.2) is vacuously satisfied. However, and in contrast with the algebraic cases, cotensors will now have the tendency of not living in $\mathscr{A}$, and the functors $\mathscr{A} \stackrel{\|}{\longrightarrow} \mathscr{U}$ will probably have a right adjoint (instead of the left adjoint that free structures provide in the algebraic case). This requires some changes in (3.3). More specifically, cotensors may be computed in $\mathscr{U}$ and then reflected into $\mathscr{A}$. This is notably the case of the full subcategory $\mathscr{A}$ of uc-generated uniform spaces (see Definition (1.6)).

\section{The enriched category of locally convex algebras}

Let us recall that a locally convex topological algebra over $F$, the real or the complex field, is an $F$-algebra $A$ with a locally convex topology making the product of $A$ separately continuous. Any such structure determines a uniformity (by the distances defined by the continuous seminorms). It is well known that continuous linear multiplicative maps $A \stackrel{f}{\longrightarrow} B$ between locally convex algebras $A$ and $B$ are automatically u-continuous. Therefore, the category $\mathscr{A}$ of locally convex topological algebras with 1 and identity preserving continuous multiplicative linear maps (abbreviated: morphisms of $\mathscr{A}$ ) is provided with a faithful forgetful functor $\mathscr{A} \stackrel{\|}{\longrightarrow} \mathscr{U}$ into the category of uniform spaces. Thus it satisfies the hypotheses of Proposition (3.1). Moreover, given any quasitopological space $X$, pointwise operators make the uniform space $\mathscr{C}(X,|A|)$ into a locally convex topological algebra with identity, and the condition (ii) (or (ii')) in (3.2) is obviously satisfied. Therefore:

(4.1) THEOREM. The category $\mathscr{A}$ of locally convex algebra with 1 is a cotensored Q-category in such a way that the forgetful functor $\mathscr{A} \stackrel{\amalg}{\longrightarrow} \mathscr{U}$ preserves cotensors and the map $\mathscr{A}(A, B) \longrightarrow \mathscr{U}(|A| B \mid$,$) is an initial map in 2$. Further if $A$ is commutative (respectively, locally m-convex (see Michael (1952)), Hausdorff, complete) then so is $\mathscr{C}(X, A)$ for any $X$ in $\mathscr{Q}$.

Proof. Define $\mathscr{C}(X, A)$ to be the uniform space $\mathscr{C}(X,|A|)$ with the algebraic structure of pointwise operations. Consider now the family of u-continuous maps

$$
|\mathscr{C}(X, A)| \stackrel{\mathscr{C}(\alpha, A)}{\longrightarrow}|\mathscr{C}(K, A)|, \quad \alpha \text { admissible. }
$$


Clearly all $\mathscr{C}(\alpha, A)$ preserve the algebraic structures and since the uniformity on $\mathscr{C}(X,|A|)$ is initial for this family (Definition (2.4)) it is locally convex (respectively, locally m-convex) if each $\mathscr{C}(K, A)$ is. But of course $\mathscr{C}(K, A)=\mathscr{U}_{u}(K, A)$, and this is a very well-known factor. Finally, since condition (ii') in Definition (3.2) is satisfied, Corollary (3.4) applies. The cases of $A$ Hausdorff or complete follow from Remarks 2 and 3 to Definition (2.4).

Suppose now that $A \stackrel{\tau_{\lambda}}{\longrightarrow} A_{\lambda}$ is a filtered diagram (see MacLane (1971), Chapter IX) in $\mathscr{A}$ and $A \stackrel{f}{\longrightarrow} B$ is a morphism in $\mathscr{A}$ with $B$ a normed algebra. If $B \stackrel{p}{\longrightarrow} \mathbf{R}^{+}$denotes the norm of $B$, the composite $p \circ f$ is a continuous seminorm $A \stackrel{p \circ f}{\longrightarrow} \mathbf{R}^{+}$of $A$, and since the diagram is filtered, there exists a $\lambda$ and a continuous seminorm $A_{\lambda} \stackrel{p_{\lambda}}{\longrightarrow} \mathbf{R}^{+}$such that $p \circ f \leqq p_{\lambda} \circ \pi_{\lambda}$. If $\pi_{\lambda}$ is surjective, we can define $A_{\lambda} \stackrel{f_{\lambda}}{\longrightarrow} B$ by $f_{\lambda}(x)=f(a)$ where $a \in A$ is any element such that $\pi_{\lambda}(a)=x$, clearly $f=f_{\lambda} \circ \pi_{\lambda}$. We illustrate the situation by the diagram

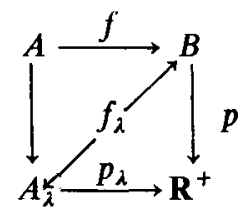

It is of course quite obvious that $f_{\lambda}$ is well defined and that it preserves the algebraic structure. Also, since $p f_{\lambda} \pi_{\lambda}=p f \leqq p_{\lambda} \pi_{\lambda}$ and $\pi_{\lambda}$ is surjective, it follows that $p f_{\lambda} \leqq p_{\lambda}$, whence $f_{\lambda}$ is continuous. Summing up: for any $A \stackrel{f}{\longrightarrow} B$ in $\mathscr{A}$ there exists a $\lambda$ and a morphism $A_{\lambda} \stackrel{f_{\lambda}}{\longrightarrow} B$ in $\mathscr{A}$ such that $f=f_{\lambda} \pi_{\lambda}$. Thus:

(4.2) Proposition. Given any filtered limit diagram $A \stackrel{\pi_{\lambda}}{\longrightarrow} A_{\lambda}$ in $\mathscr{A}$ with surjective $\pi_{\lambda}$ for all $\lambda$, and any normed algebra $B$, the diagram

$$
\mathscr{A}\left(A_{\lambda}, B\right) \stackrel{\mathscr{A}\left(\pi_{\lambda}, B\right)}{\longrightarrow} \mathscr{A}(A, B)
$$

is a filtered colimit diagram of quasitopological spaces.

Proof. It suffices (by (0.0)) to show that given any admissible $K \stackrel{\alpha}{\longrightarrow} \mathscr{A}(G, B)$ there exists an admissible $K \stackrel{\alpha_{\lambda}}{\longrightarrow} \mathscr{A}\left(A_{\lambda}, B\right)$ such that $\alpha=\mathscr{A}\left(\pi_{\lambda}, B\right) \circ \alpha_{\lambda}$. We do this as follows: by (4.1), the corresponding function $A \stackrel{\gamma}{\longrightarrow} \mathscr{C}(K, B)$ (where $(\gamma(a))(k)=(\alpha(k))(a))$ is a morphism in $\mathscr{A}$. But for $K$ compact and $B$ normed, clearly $\mathscr{C}(K, B)\left(=\mathscr{U}_{u}(K, B)\right)$ is also normed, and according to the observations above, there exists $\lambda$ and $A_{\lambda} \stackrel{\gamma_{\lambda}}{\longrightarrow} \mathscr{C}(K, B)$ with $\gamma=\gamma_{\lambda} \pi_{\lambda}$. This shows that for the corresponding admissible $K \stackrel{\alpha_{\lambda}}{\longrightarrow} \mathscr{A}\left(A_{\lambda}, B\right)$ (that is, $\left(\alpha_{\lambda}(k)\right)(a)=\left(\gamma_{\lambda}(a)\right)(k)$ ) we have $\alpha=\mathscr{A}\left(\pi_{\lambda}, B\right) \circ \alpha_{\lambda}$. This concludes the proof. 
Recall that $F$ denotes either the real field or the complex field. According to the definitions, for any quasitopological space $X$, we have that $\mathscr{C}(X, F)$ is just the algebra of continuous scalar valued functions with the uniform convergence on admissible maps, that is: a defining family of seminorms for $\mathscr{C}(X, F)$ is given by the $p_{\alpha}(f)=\sup \{\mid f(\alpha(k)) ; f \in K\}$ for $K \stackrel{\alpha}{\longrightarrow} X$ admissible.

EXAMPLE. Let $X$ be any Hausdorff topological space. An admissible map $K \stackrel{\alpha}{\longrightarrow} X$ (which is just a continuous function) has compact image. Therefore a morphism $X \longrightarrow q F$ in $\mathscr{Q}$ is exactly a function $X \longrightarrow F$ which is continuous on the compact subsets of $X$, and $\mathscr{C}(X, F)$ is the algebra of these functions with the uniform convergence on the compact subsets of $X$. Such algebras have been studied long since (see Michael (1952) and Dubuc and Porta (1971)).

Just as in the case of the example above, we can easily prove (using (4.1)):

(4.3) Proposition. For any quasitopological space $X$, the algebra $\mathscr{C}(X, F)$ is a commutative Hausdorff complete locally m-convex algebra with 1 . Moreover, when $F=\mathbf{C}$ the complex numbers, $\mathscr{C}(X, \mathbf{C})$ is a pro- $C^{*}$-algebra.

Recall that a pro- $C^{*}$-algebra (see Bourdaud (1975/6), Michael (1952)) is a commutative complete, Hausdorff, locally m-convex complex algebra $A$ with an involution $x \longrightarrow x^{*}$ and a defining family of seminorms $\left\{p_{i}\right\}$ such that

$$
p_{i}\left(x x^{*}\right)=\left(p_{i}(x)\right)^{2} \text { for all } x \in A \text { and all } p_{i} \text {. }
$$

The algebras $\mathscr{C}(X, \mathbf{C})$ are obviously pro- $C^{*}$-algebras with $f^{*}=$ complex conjugate of $f$ and the seminorms $p_{\alpha}$ defined above.

Let now $A$ be any algebra in $\mathscr{A}$. The quasitopological space $\mathscr{A}(A, F)$ is just the set of continuous characters $A \stackrel{\varphi}{\longrightarrow} F$ (with a certain quasitopology), that is, the spectrum (or 'carrier space') of $A$ (see Michael (1952), Dixmier (1964), Bourbaki (1967)). But contrary to the traditional treatment, we will not endow it with the pointwise convergence (that would be the space $\mathscr{A}_{n}(A, F)$ ) but with the quasitopology resulting from the forgetful functor $A \longrightarrow|A|$ into $\mathscr{U}$. More precisely:

(4.4) Definition. For any algebra $A$ in $\mathscr{A}$, the spectrum of $A$ is the quasitopological space $\mathscr{A}(A, F)$.

As observed above, $\mathscr{A}(A, F)$ has the same underlying set as the traditional $\mathscr{A}_{\pi}(A, F)$ and the identity map $\mathscr{A}(A, F) \longrightarrow \mathscr{A}_{\pi}(A, F)$ is continuous by Definition (2.2).

EXAMPLE 1. If $A$ is a Fréchet algebra any compact subset of $\mathscr{A}_{\pi}(A, F)$ is 
u-equicontinuous (see Michael (1952)). Therefore any continuous $K \stackrel{\alpha}{\longrightarrow} \mathscr{A}_{\pi}(A, F)$ is admissible for $\mathscr{A}(A, F)$. Thus, the identity $\mathscr{A}(A, F) \stackrel{\text { id }}{\longrightarrow} q \mathscr{A}_{\pi}(A, F)$ is bicontinuous. In other words, the quasitopology of $\mathscr{A}(A, F)$ is the quasitopology of the classical spectrum. More particularly, if $A$ is a normed algebra in $\mathscr{A}$, the space $\mathscr{A}_{\pi}(A, F)$ is compact (and uniformly equicontinuous), and according to the agreement of not writing $q$ in this case (first paragraph of Section 1) we have then $\mathscr{A}(A, F)=\mathscr{A}_{\pi}(A, F)$ for $A$ normed with identity.

EXAMPLE 2. The identity $\mathscr{A}(A, F) \longrightarrow \mathscr{A}(A, F)$ induces a map

$$
A \stackrel{j}{\longrightarrow} \mathscr{C}(\mathscr{A}(A, F), F)
$$

(that we study below in detail under another name). The initial uniformity on $A$ induced by $j$ and $\mathscr{C}(\mathscr{A}(A, F), F)$ need not coincide with that of $A$. The topology of this uniformity is the topology $\tau_{0}(A)$ of Michael (see Michael (1952), p. 32). The reason is clear, since $\tau_{0}(A)$ is the topology of uniform convergence on u-equicontinuous compact subsets of $\mathscr{A}_{\pi}(A, F)$.

(4.4) Proposition. The spectrum $\mathscr{A}(A, F)$ of any locally convex algebra $A$ is a u-separated quasitopological space.

Proof. From Remark 1 to (2.3) we get that $\mathscr{U}(|A|,|F|)$ is u-separated. Now use Corollary (1.10)(b) to conclude that $\mathscr{A}(A, F) \hookrightarrow \mathscr{U}(|A|,|F|))$ is also u-separated.

According to the remark at the end of Example 1 above, for each commutative $C^{*}$-algebra $C$ we get that the quasitopological space $\mathscr{A}(C, F)$ is compact. Similarly, by definition, for each $K$ compact the cotensor $\mathscr{C}(K, F)$ is a commutative $C^{*}$ algebra if $F$ is the complex field. Therefore, the classical Gelfand duality between compact spaces and $C^{*}$-algebras applies here to give (see Dixmier (1964), Bourbaki (1967)):

(4.5) Proposition.

(a) For any compact space $K$, the map $K \stackrel{\wedge}{\longrightarrow} \mathscr{A}(\mathscr{C}(K, F), F), \hat{k}(f)=f(k)$ is a bicontinuous bijection.

(b) If $F$ is the complex field and $C$ a commutative $C^{*}$-algebra with 1, the map $C \stackrel{\wedge}{\longrightarrow} \mathscr{C}(\mathscr{A}(C, F), F), c(f)=f(c)$ is an isometric *-isomorphism.

Consider now the $\mathscr{2}$-category $\mathscr{A}$ and the pair of $\mathscr{2}$-adjoint functors:

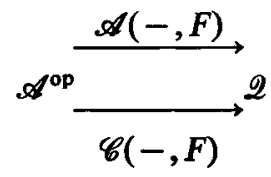


As in any 2 -adjointness, both composites

$$
G=\mathscr{C}(\mathscr{A}(-, F), F) \quad \text { and } \quad D=\mathscr{A}(\mathscr{C}(-, F), F)
$$

are 2-monads (sometimes called 2-triples; see MacLane (1971), Schubert (1972) or Dubuc (1970) for the enriched case) with units $A \stackrel{\eta A}{\longrightarrow} G A$ and $X \stackrel{d X}{\longrightarrow} D X$ given by the formulas $\eta A(a)(\varphi)=\hat{a}(\varphi)=\varphi(a)$ and $d X(x)(f)=\hat{x}(f)=f(x)$. This follows from (4.1) and (2.5), Remark 1.

(4.6) Definition. The functor $\mathscr{A} \stackrel{G}{\longrightarrow} \mathscr{A}$ will be called the Gelfand $\mathscr{Q}$-monad, and its unit $\eta A$ the Gelfand transformation. The functor $\mathscr{Q} \stackrel{D}{\longrightarrow} \mathscr{Q}$ will be called the Dirac 2-monad, and its unit $d X$ the Dirac transformation.

Our next goal is to extend the Gelfand duality of (4.5) to this context. In fact (4.5) just means that $D K=K$ for all compact $K$ and (assuming that $F$ is the complex field) $G A=A$ for $A$ a commutative $C^{*}$-algebra. We will identify the largest class of algebras for which $G A=A$ and the largest class of quasitopologies for which $D X=X$.

(4.7) Proposition. A quasitopological space $X$ is $u$-separated if and only if the Dirac transformation $X \stackrel{d X}{\longrightarrow} D X=\mathscr{A}(\mathscr{C}(X, F), F)$ is a bicontinuous bijection.

Proof. Suppose that $X$ is u-separated (Definition (1.8)) and let $K \stackrel{\alpha}{\longrightarrow} X$ be a filtered colimit diagram in $\mathscr{Q}$ such that all the $\alpha$ 's are injective admissible maps with the $F$-valued extension property (Proposition (1.13)). Using Remark 2 to (2.5) we get that $\mathscr{C}(X, F) \stackrel{\mathscr{C}(\alpha, F)}{\longrightarrow} \mathscr{C}(K, F)$ is a filtered diagram of locally convex algebras. Since all $\mathscr{C}(\alpha, F)$ are surjective functions (by (1.12)), Proposition (4.2) applies to give that

$$
\mathscr{A}(\mathscr{C}(K, F), F) \stackrel{\mathscr{A}(\mathscr{C}(\alpha, F), F)}{\longrightarrow} \mathscr{A}(\mathscr{C}(X, F), F)
$$

is a filtered colimit diagram. Consider now

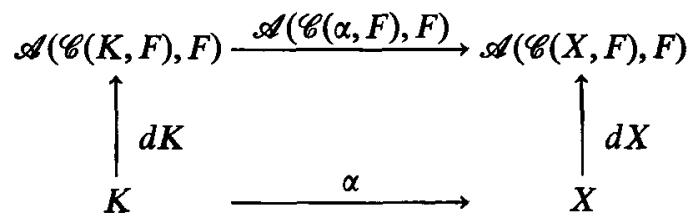

Both horizontal subdiagrams are filtered colimit diagrams, and since each $d K$ is a bicontinuous bijection by (4.5)(a), it follows that $d X$ is a bicontinuous bijection. 
Conversely, if $d X$ is a bicontinuous injection, it follows from (4.4) that $X$ is u-separated. This ends the proof.

Thus, the u-separated quasitopologies are exactly the spectra of locally convex algebras. This generalizes (7.6) in Michael (1952).

From (4.7) we get also:

(4.8) Proposition.

(a) For any locally convex algebra $A$ in $\mathscr{A}$, the Dirac transformation

$$
d: \mathscr{A}(A, F) \longrightarrow D \mathscr{A}(A, F)
$$

is a bicontinuous bijection of quasitopologies.

(b) For any quasitopological space $X$ in $\mathscr{Q}$, the Gelfand transformation

$$
\eta: \mathscr{C}(X, F) \longrightarrow G \mathscr{C}(X, F)
$$

is a bicontinuous isomorphism of locally convex algebras.

Proof. A simple categorical computation shows that (a) holds if and only if (b) holds. Now, from (4.4) we get that $\mathscr{A}(A, F)$ is u-separated and so (a) follows from (4.7).

(4.9) CoRollary. Both the Gelfand 2-monad $G$ and the Dirac 2-monad $D$ are idempotent: $G G=G$ and $D D=D$. More precisely, for each $A$ in $\mathscr{A}$,

$$
G A \stackrel{\eta G A}{\longrightarrow} G G A \text { and } D X \stackrel{d D X}{\longrightarrow} D D X
$$

are isomorphisms.

We consider next the 2-category of G-algebras (Dubuc (1970), MacLane (1971), Schubert (1972)). Since $G$ is idempotent, the $G$-algebras form the full $\mathcal{Q}$-subcategory of $\mathscr{A}$ whose objects are the locally convex algebras $A$ for which there is a left inverse $G A \stackrel{\xi}{\longrightarrow} A$ for the Gelfand transform $A \stackrel{\eta A}{\longrightarrow} G A$, that is, $\xi \circ \eta A=\mathrm{id}$. It follows (from categorical arguments) that $\xi$ is also a right inverse, so that $\eta A \circ \xi=$ id, and $\eta A$ is a bicontinuous isomorphism. Identical considerations apply to the Dirac 2 -monad in $\mathscr{Q}$. In view of this, the result in (4.7) amounts to identifying the quasitopologies that are $D$-algebras as being precisely the u-separated quasitopologies.

For the Gelfand $\mathscr{Q}$-monad we have:

(4.10) Proposition. A complex locally convex algebra with identity is a G-algebra if and only if it is a pro- $C^{*}$-algebra (cf. paragraph after (4.3)). 
Proof. Clearly (4.3) applies to give one of the implications. Suppose then that $A$ is a pro- $C^{*}$-algebra with identity. Any symmetric continuous seminorm $A \stackrel{p}{\longrightarrow} \mathbf{R}_{+}$determines a $C^{*}$-algebra $\hat{A}(p)$ ( = completion of the quotient of $A$ by the null space of $p$ ), and $A \longrightarrow \widehat{A}(p)$ ( $p$ an arbitrary symmetric continuous seminorm) is a limit diagram. Now from the classical result (4.5)(b), we get that each $\widehat{A}(p)$ is a $G$-algebra. Hence, since any limit of $G$-algebras is a $G$-algebra, it follows that $A$ is, as desired.

RemarK 1. Recall that the condition that $\eta A$ be injective is equivalent to $A$ being semisimple, but this is weaker than the requirement that $A$ be a $G$-algebra.

Remark 2. Proposition (4.10) above contains the equivalence $(a) \Leftrightarrow(e)$ in Michael (1952), Theorem 8.4.

We will denote by $\mathscr{Q}_{2}$ the 2 -category of u-separated quasitopologies (and continuous maps), and by pro- $\mathscr{C}^{*}$ the $\mathscr{2}$-category of pro- $C^{*}$-algebras with identity. These coincide with the categories of $G$-algebras and $D$-algebras, respectively.

From purely categorical arguments we get now:

\section{(4.11) THEOREM.}

(a) Assume that $F$ is the complex field. Then the 2-category pro $\mathscr{C}^{*} \hookrightarrow \mathscr{A}$ of pro-C*-algebras (commutative, with identity) is a full reflexive 2 -sub-category of $\mathscr{A}$, that is, the inclusion pro $\mathscr{C}^{*} \hookrightarrow \mathscr{A}$ has a $\mathscr{Q}$-left adjoint $\mathscr{A} \stackrel{m}{\longrightarrow}$ pro $\mathscr{C}^{*}$. For any $A$ in $\mathscr{A}, m A=\mathscr{C}(\mathscr{A}(A, F), F)$ and the unit of the reflexion $A \longrightarrow m A$ is the Gelfand transform. Further, $A$ is in pro $\mathscr{C}^{*}$ if and only if $A \cong m A$.

(b) $A$ locally convex algebra $A$ is in pro $\mathscr{C}^{*}$ if and only if it is the algebra $\mathscr{C}(X, F)$ of all continuous complex-valued functions on a quasitopological space $X$ with the uniform convergence on admissible maps (and there is a unique u-separated such $X$ : the spectrum of $A$ ).

(c) Let $F$ denote now either the real or the complex field. The 2 -category $\mathscr{2}_{2} \hookrightarrow \mathscr{2}$ of $u$-separated quasitopologies is a full reflexive 2-subcategory of 2. That is, the inclusion $\mathscr{Q}_{2} \hookrightarrow \mathscr{Q}$ has a $\mathscr{Q}$-left adjoint $\mathscr{Q} \stackrel{\leftrightarrow}{\longrightarrow} \mathscr{Q}_{2}$. For any $X$ in $\mathscr{Q}$,

$$
u X=\mathscr{A}(\mathscr{C}(X, F), F)
$$

and the unit of the reflexion $X \longrightarrow u X$ is the Dirac transform. Further, $X$ is in $\mathscr{Q}_{2}$ if and only if $X \cong u X$.

(d) A quasitopological space $X$ is u-separated if and only if it is the spectrum of some locally convex algebra $A$ (and there is a unique such $A$ in pro $\mathscr{C}^{*}$ : the algebra $\mathscr{C}(X, F))$.

(e) Assume again that $F$ is the complex field. The categories pro $\mathscr{C}^{*}$ and $\mathscr{Q}_{2}$ are 
dual categories:

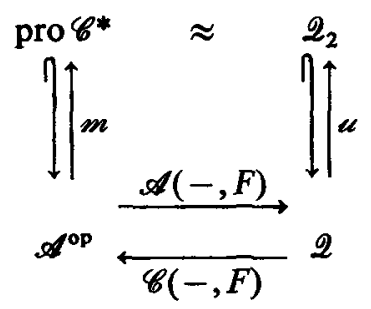

The equivalence on the upper half of this diagram is given by the restrictions of the 2-functors of the lower half.

We close this paper with two propositions that supply additional information about the Dirac and Gelfand transforms.

(4.12) Proposition. For any quasitopological space $X$ the Dirac map $X \stackrel{d X}{\longrightarrow} u X$ is the surjective quotient map in 2. Thus, $u X$ is the 'largest' $u$-separated quotient of $X$.

Proof. Observe that if we factor

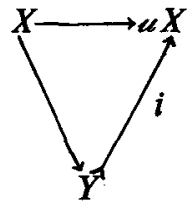

where $Y$ is a quasitopological quotient and $i$ is a continuous injective function (cf. (0.9)), then it follows from (1.10)(b) that $Y$ is u-separated. But then it is easy (using a categorical argument) to see that $Y$ satisfies the universal property of $u X$, whence $Y=u X$.

We remark that in order to compute $u X$ it is irrelevent whether we use real or complex numbers.

(4.13) Proposition. Assume that $F$ is the complex field. For any locally convex algebra $A$ with 1 the map $A \stackrel{\eta A}{\longrightarrow} m A$ is an injection if and only if $A$ is semisimple, and it is an initial injection if for all continuous seminorms $A \stackrel{p}{\longrightarrow} \mathbf{R}_{+}$, the quotient $A(p)$ of $A$ by the null space of $p$ is semisimple.

Proof. In view of Remark 1 to (4.10) we only have to establish the second part of the statement. Suppose the hypothesis is satisfied and consider the continuous functions $\mathscr{A}(A(p), F) \stackrel{\mathscr{A}\left(\pi_{p}, f\right)}{\longrightarrow} \mathscr{A}(A, F)$ where $A \stackrel{\pi_{p}}{\longrightarrow} A(p)$ is the quotient map. Since $A(p)$ is a normed algebra with 1 , the space $\mathscr{A}_{\pi}\left(A_{p}, F\right)$ is a compact space and $\mathscr{A}(A(p), F)=\mathscr{A}_{\pi}(A(p), F)$ (see Example 1, following Definition 
(4.4)). Therefore the map $\mathscr{A}\left(\pi_{p}, F\right)$ is an admissible map. Let now $\left\{a_{\lambda}\right\}$ be any net in $A$ and suppose that $\hat{a}_{\lambda} \longrightarrow 0$ in $m A$. Given any $p$, it follows that

$$
\hat{a}_{\lambda}\left(\mathscr{A}\left(\pi_{p}, F\right)(\varphi)\right) \longrightarrow 0
$$

for all continuous characters $A(p) \stackrel{\varphi}{\longrightarrow} F$. Then $\varphi\left(\pi_{p}\left(a_{\lambda}\right)\right) \longrightarrow 0$ for all $\varphi$. But since $A(p)$ is a semisimple normed algebra, we conclude that $\pi_{p}\left(a_{\lambda}\right) \longrightarrow 0$, or $p\left(a_{\lambda}\right) \longrightarrow 0$ and this means that $a_{\lambda} \longrightarrow 0$ in $A$. Hence convergence in $A$ coincides with convergence in $m A$.

\section{References}

R. Arens (1946), 'A topology for spaces of transformations', Ann. of Math. (2) 47, 480-495.

E. Beckenstein, L. Narici and C. Suffel (1977), Topological algebras (Notas de Matemática No. 24, North-Holland, Amsterdam).

G. Bourdaud (1975/6), 'Sur la dualité des algèbres localement convexes', C. R. Acad. Sci. Paris 281, 1011-1014; 282, 313-316.

N. Bourbaki (1940), Eléments de mathématiques, Topologie générale (Hermann, Paris).

N. Bourbaki (1967), Éléments de mathématiques, Thèories spectrales (Hermann, Paris).

A. Clark (1969), 'Quasitopologies and compactly generated spaces' Ph.D. Dissertation.

B. Day (1972), 'A reflexion theorem for closed categories', J. Pure Appl. Algebra 2, 1-11.

J. Dixmier (1964), Les $C^{*}$-algèbres et leurs réprésentations (Gauthier-Villars, Paris).

E. J. Dubuc (1970), Kan extensions in enriched category theory (Lecture Notes No. 145, SpringerVerlag, Berlin).

E. J. Dubuc (1977), 'Concrete quasitopoi', Proc. Durham Conference (Springer-Verlag, Berlin) (to appear).

E. J. Dubuc and H. Porta (1971), 'Convenient categories of topological algebras and their duality theory', J. Pure Appl. Algebra 1', 281-316.

S. Eilenberg (1969), Spanier quasitopologies (Lecture notes, University of Paris).

S. Ginsburg and J. R. Isbell (1959), 'Some operators on uniform spaces', Trans. Amer. Math. Soc. 93, 145-168.

D. Hsia (1959), 'Seminormed Banach rings with involutions', Izv. Akad. Nauk SSSR, Ser. Mat. 23, 509-528 (Russian).

J. R. Isbell (1964), Uniform spaces (Math. Surveys No. 2 A.M.S., Providence).

J. Kelley (1955), General topology (Van Nostrand, New York).

W. F. Lamartin (1977), On the foundations of k-group theory, Dissertationes Mathematica, CXLVI (Polska. Akad. Nauk, Warszawa).

M. C. McCord (1970), 'Classifying spaces and infinite symmetric products', Trans. Amer. Math. Soc. 146, 273-298.

S. MacLane (1971), Categories for the working mathematician (Springer-Verlag, Berlin).

E. Michael (1952), 'Locally multiplicatively-convex topological algebras', Amer. Math. Soc. Memoirs 11.

H. Schubert (1972), Categories (Springer-Verlag, Berlin).

E. Spanier (1959), 'Infinite symmetric products, function spaces and duality', Ann. of Math. 69, $142-198$.

E. Spanier (1962), 'Quasitopologies', Duke Math. J. 30, 1-14.

University of Illinois at Urbana-Champaign

Urbana

Illinois 61801

U.S.A.
Facultad de Ciencias Exactas Universidad de Buenos Aires

Buenos Aires

Argentina 